\title{
ESTABLISHING AN APPROPRIATE LEVEL OF DETAIL (LOD) FOR A BUILDING INFORMATION MODEL (BIM) - WEST BLOCK, PARLIAMENT HILL, OTTAWA, CANADA
}

\author{
S. Fai ${ }^{\text {a }}$, J. Rafeiro ${ }^{\text {a }}$
}

\author{
${ }^{\text {a }}$ Carleton Immersive Media Studio (CIMS), Azrieli School of Architecture and Urbanism, Carleton University, 1125 \\ Colonel By Drive, Ottawa, Canada K1S 5B6 \\ sfai@cims.carleton.ca \\ jesse.rafeiro@carleton.ca
}

KEY WORDS: building information modelling, level of detail, parametric modelling, Gothic Revival architecture,

\begin{abstract}
:
In 2011, Public Works and Government Services Canada (PWGSC) embarked on a comprehensive rehabilitation of the historically significant West Block of Canada's Parliament Hill. With over 17 thousand square meters of floor space, the West Block is one of the largest projects of its kind in the world. As part of the rehabilitation, PWGSC is working with the Carleton Immersive Media Studio (CIMS) to develop a building information model (BIM) that can serve as maintenance and life-cycle management tool once construction is completed. The scale and complexity of the model have presented many challenges. One of these challenges is determining appropriate levels of detail (LoD). While still a matter of debate in the development of international BIM standards, $\mathrm{LoD}$ is further complicated in the context of heritage buildings because we must reconcile the LoD of the BIM with that used in the documentation process (terrestrial laser scan and photogrammetric survey data). In this paper, we will discuss our work to date on establishing appropriate LoD within the West Block BIM that will best serve the end use. To facilitate this, we have developed a single parametric model for gothic pointed arches that can be used for over seventy-five unique window types present in the West Block. Using the AEC (CAN) BIM as a reference, we have developed a workflow to test each of these window types at three distinct levels of detail. We have found that the parametric Gothic arch significantly reduces the amount of time necessary to develop scenarios to test appropriate LoD.
\end{abstract}

\section{INTRODUCTION}

\subsection{Context}

"There is, I repeat, no degradation, no reproach in this, but all dignity and honourableness: and we should err grievously in refusing either to recognize as an essential character of the existing architecture of the North, or to admit as a desirable character in that which it yet may be, this wildness of thought, and roughness of work; this look of mountain brotherhood between the cathedral and the Alp; this magnificence of sturdy power, put forth only the more energetically because the fine finger-touch was chilled away by the frosty wind, and the eye dimmed by the moor-mist, or blinded by the hail; this outspeaking of the strong spirit of men who may not gather redundant fruitage from the earth, nor bask in dreamy benignity of sunshine, but must break the rock for bread, and cleave the forest for fire, and show, even in what they did for their delight; some of the hard habits of the arm and heart that grew on them as they swung the axe or pressed the plough."

John Ruskin The Stones of Venice

Built on a rocky outcrop overlooking the turbulent Ottawa River, Canada's Parliament Hill is a testament to the "savageness" of John Ruskin's Gothic Architecture. Framed by three monumental buildings — the West, Centre and East Blocks - "The Hill" is a designated National Historic Site and both the political and symbolic heart of the country.
Historically, the West Block has housed committee rooms and offices for parliamentarians - including the Prime Minster. The building that we see today was designed in three phases Construction began in 1859, following the Gothic Revival design of Thomas Stent and Augustus Laver. Construction of the first phase was completed in 1866. The second and third phases, also Gothic in design, were completed in 1878 and 1906, respectively. The building underwent a significant renovation to the interior and exterior in 1965.

\subsection{BIM and Heritage Buildings}

Although optimized for the design and construction of new buildings, modern Building Information Modelling (BIM) software is being adopted for historic buildings for a variety of purposes across Europe, including facilities management, (http://www.ebim.co.uk/) (http://www.plowmancraven.co.uk/) and cultural heritage preservation (Garagnani, S.)(Baik, A.)(Oreni, D.)(Brumana, R.). Guidelines for the standardization of BIM procedures are currently governed nationally across the world but have been receiving international collaborative efforts to develop stronger frameworks for integration into the Architectural, Engineering and Construction industry (AEC) globally by BuildingSMART (BuildingSMART.org). While these sources struggle to standardize BIM within the AEC industry, possibly an even greater challenge is to standardize BIM procedures for modelling historic buildings where likeelements differ in scale from one instance to the next (Hichri et 
al.) and where the BIM model directly corresponds to the documentation strategy employed.

In most cases of heritage BIM, the use of laser scanning provides the most accurate documentation method for which the model can be based. No software as of yet has proven adequate to directly model a building from raw TLS data, however many strategies are being explored (Dekeyser). To compensate for the inability to automate a BIM from TLS data or other documentation methods, many semi-automatic and manual modelling strategies have been explored. One group (C. Dore, M. Murphy 2013) has modelled a library of parametric classical architectural elements from historic manuscripts and architectural pattern books wherein elements from the library can be incorporated into a parametric building façade developed for fast and efficient modelling of building façades. Both the objects and facade were built within ArchiCAD and an embedded programming language called Geometric Description Language (GDL) (C. Dore, M. Murphy 2013). (M. Ludwig et al. 2013) has used a parametric modelling strategy in Revit for the reconstruction of the Church of St. Catherine in Nuremburg where parametric modelling was found to be suitable for upgrading models from lower to finer detail through the capacity to apply changes to multiple objects at the same time and found the modelling process was hastened by the ability for similar shapes to be derived from one another. The group used a total station survey, photogrammetric survey, drawings, and footprints of the building (M. Ludwig et al. 2013). This method, compared to those previously mentioned is most similar to the West Block BIM in terms of the data used and the modelling strategy however no attention is given to how the LoD of the model is defined.

\subsection{Related Works}

The following section introduces the past work of several groups that have used parametric modelling techniques for modelling historic architecture in applications other than BIM software. Very few examples of models exist which are comparable in scale and variability to the modelling of the West Block so it is valuable to introduce other strategies of modelling as a means to compare. These examples, like the West Block, have engaged strategies to hasten modelling, support non-linear modelling methods and reduce the amount of modelling required by establishing parametric controls for modelled elements. One group (Havemann, Fellner 2004) has formalized the modelling process of prototypical gothic window constructions with a Generative Modelling Language (GML) by conjoining a series of basic geometric patterns together with a style library. Another group (Chevrier et al. 2010) produced parametric models of common architectural openings found in heritage structures of Montreal, Canada and Nancy, France within Maya Environment and also developed a graphical user interface (GUI) to assist users in applying modifications using point cloud, 2D plans, or photographs. The method employed by the above examples are innovative in that their approach involves first the modelling of typical or generic elements and to then later adapt these elements to specific instances of precisely measured data. The former strategy while innovative for its ability to produce a wide variety of gothic windows within a compact representation method is a strategically viable approach to modelling. (Boulaassal, H. et al. 2010) devised a workflow for parametric modelling involving Terrestrial Laser Scanning data and the use of segmentation algorithms, automatic edge extraction, intersection point calculations and finally the development of a graphical user interface to construct and accurately size parametric objects such as openings into their correct position. While innovative for their approach, these modelling methods cannot be reproduced within the AEC's most popular BIM software such as Autodesk Revit. The project management and strategies applied are however very relevant to the research.

\subsection{Types of Gothic Arches and the Gothic Revival style of the West Block}

The architectural period defined as Gothic was a "continuous logical development of the Romanesque movement," which dates from the mid- $12^{\text {th }}$ century to the end of the 15 th century (Corroyer, 1893). One defining characteristic of Gothic architecture is the elaborate use of the pointed arch for structural efficiency and functional purposes such as window and door openings. In the portfolio of Villard de Honnecourt, written sometime during the 13th century, a series of instructions for drawing and designing arches are presented and accredited to the development of gothic architecture (Carl F. Barnes, Jr.). (Bloxam, 1882) defines the progression of English ecclesiastical architecture into seven periods starting from the 11 th century to the early 17 th century according to distinguishable forms of arches and manner of ornament which has evolved into many forms dating from the roman times until the fifteen century (Bloxam, 1882).
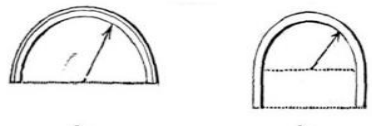

ז.

2.
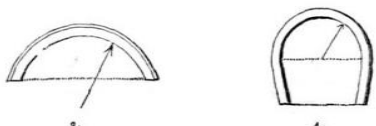

$$
4 .
$$
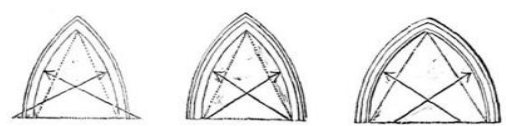

5.

6.
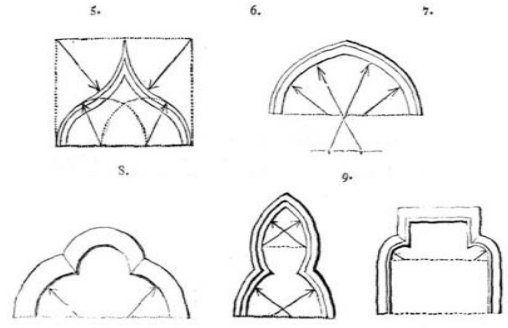

rs.

Figure 1. Several different types of arches used in gothic architecture (Bloxam, 1882). Arches 1-4 are classified as round-headed arches and consist of the semicircular arch (1), stilted arch (2), segmental arch (3) and horse-shoe arch (4). Arches 5-7 are classified as pointed arches and consist of the Lancet pointed arch (4), Equilateral pointed arch (5) and the Obtuse-angled pointed arch (6). Arches 8-9 are classified as complex pointed arches and consist of the Ogee arch (7), Tudor arch (8). Lastly arches 10-12 are referred to as foiled arches and consist of the roundheaded trefoil (10), the pointed trefoil (11) and the square headed trefoil (12). 
In the 1830 s the principles of Gothic architecture were reintroduced by a style known as Gothic Revival which at the time had become Britain's national style for which Canada would attain inspiration for the design of their parliament (C.A. Young 1995). The approach of Gothic Revival architects according to (C. A. Young 1995) "was to select elements from all parts of the gothic past and combine them with the materials and needs of the present." The original structure of the West Block completed in 1865 was an "L" shaped building with two wings facing the East and the South, the dominant arch used in the openings of these faces were pointed arches such as the Lancet, Equilateral and Obtuse-angled pointed arch. Later in 1878 the Mackenzie Tower and the Mackenzie Wing to the West would be completed in 1878 wherein the most commonly used arches were round-headed arches such as the Semicircular arch, Stilted arch, and Segmental arch. In 1909 the final construction comprising of the Laurier tower and wing to the North was completed, with arched openings primarily consisting of a combination of complex pointed arches such as the Ogee arch, Tudor arch and some instances of round-headed arches.

\section{MODELLING PROCESS}

Since the use of the BIM was not fully known at the outset of the project, the modelling strategy remained as flexible as possible in terms of the ability to easily increase or reduce the LoD of the modelled elements to suit the needs of the BIM's future users. Research into current definitions and standards for BIM LoD was conducted to better understand how LoD relates to the intended use of a BIM. Typically $\mathrm{LoD}$ is defined as a progression of a model's graphic representation beginning with a generic $2 \mathrm{D}$ representation at the lowest $\mathrm{LoD}$ to various amounts of graphic or non-graphic information attached to 3D modelled objects; examples of non-graphic information are fabrication, assembly and relationship of the object to other modelled objects of the BIM (usually accepted as the highest LoD) (BIMForum; American Institute of Architects; Autodesk workshop; AEC CAN BIM; BIMtaskgroup). In the AEC industry, levels of detail will fluctuate during the design project depending on the amount of information acquired. Understandably as the project nears completion the model becomes more detailed as more information has been obtained (BIM FORUM). While a high LoD provides the most information, it is not necessarily ideal to have a high LoD in all modelled elements. On the Autodesk Sustainability Workshop website it is explained that the highest level of detail, LOD 500, used for operations and maintenance needs, "less detail than LOD 400 models so that critical information can be accessed quickly and easily," (Autodesk Sustainability Workshop). The consequence of a high LoD is an increased file size and subsequently a slowly operating BIM. Since LoD has generally been established as a method for translating project information pertinent to phases of design and construction into a BIM, defining LoD in the case of the West Block BIM has been explored as a means to propose a variety of LoDs to the future users of the model for feedback in what types of information are most useful within the model or best kept as information elsewhere. For the West Block BIM, the use of the Canadian BIM protocol (AEC CAN BIM) was used as a reference document to assist in defining three $\mathrm{LoD}$.

The BIM in its entirety has been modelled from a diverse set of data including historical drawings, 2D CAD drawings, 2D tracings in CAD from a photogrammetric survey of the exterior facades, total station surveys of the roof and interior courtyard, a series of geo-referenced terrestrial laser scans (TLS) of the interior and exterior and photographs of the interior and exterior. The sources of data for the model were produced and delivered by the Heritage Conservation Directorate (HCD) and Public Works and Government Services Canada (PWGSC). The 2D photogrammetric survey tracings, total station survey and the TLS data (as a point cloud in .pts format) were imported directly into Autodesk Revit 2014 and used to model and position the walls and all architectural objects. The primary source of data used to model the basic walls and elements was the point cloud. The point cloud, of all sources provides enough metric information required, in most cases to define basic geometry and model detailed architectural elements.

Of the aforementioned data sources, the $2 \mathrm{D}$ photogrammetric tracings, point cloud and photographs of the interior and exterior as references were the primary sources used in modelling the stone arch openings. The modelling of the parametric openings was accomplished with Revit families. Initially two generic models were produced, and from these models, a variety of typical models to represent each opening type was made. The typical model could then be placed into multiple instances of the same opening type and even parametrically in cases where multiple instances of the same opening type varied in scale. The $2 \mathrm{D}$ photogrammetric tracings were an integral resource used to create the typical, parametric models of each opening type at their approximate scales. Together with reference to photographs, the tracings also assisted in assigning basic material values to the modelled elements at all levels of detail. With these typical, parametric models, the point cloud was used to correctly size and place the openings within the modelled walls by adjusting the numeric parametric values. At the highest level of detail, individual tracings of each opening were used to produce very specific and accurate models of each opening which record the variations of the stone tracery across each opening.

\subsection{Establishing Parameters}

Early in the modelling process attempts were made to reduce the amount of modelling and number of models necessary to create the Gothic arch openings. It was discovered that the majority of rounded and pointed arch opening types present within the West Block could be reduced to a single parametric model by carefully setting and establishing reference planes and reference lines within the Revit family modeller (Figure 2 ). The same parametric modelling procedure was then also applied to the square-headed trefoil opening types. From these two models over 75 different types of openings present within the West Block could be generated (Figure 3). Along with the 2D tracings of the photogrammetric survey, the two parametric models serve as generic templates for further modelling each opening type as a separate family. These opening type families are capable of further increase in LoD when teamed with the $2 \mathrm{D}$ tracings and point cloud since additional geometry can be mapped to the data and fixed to the original established parameters.

The parameters established define how far the centre of the window frame is from the exterior face of the wall, as well as the height and width of the opening, height of the arch and the radius of the arch (Figure 4). The opening itself is composed of a series of void blends whose profiles are locked to the established parameters. The void blends depict the scale of the exterior, interior and central measurements of the opening. The addition or subtraction of additional sets of parametric voids to 
the template model was sometimes required to compensate opening types which composed of additional geometry. In some cases for instance, a parametric circular void needed to be added to the template model. In any case the initial families shown in figure 2 are repeated across a substantial majority of the opening types. Even in cases where two or three arches are present in a single opening type, the template model can be nested into a new family multiple times, retaining its parametric functionality.
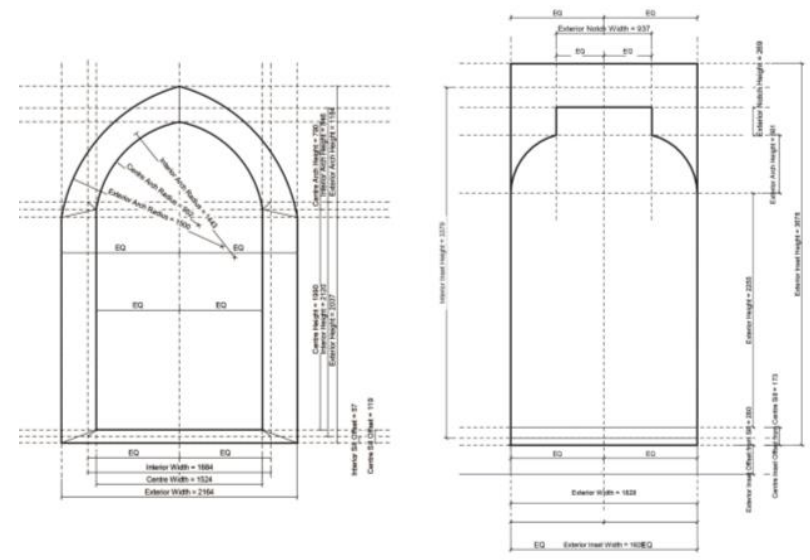

Figure 2. Reference planes and all associated parameters of two generic opening families. The family on the left can be used to produce all of the round-headed and pointed arch opening types, the family on the right can be used to produce all of the square-headed trefoil opening types.

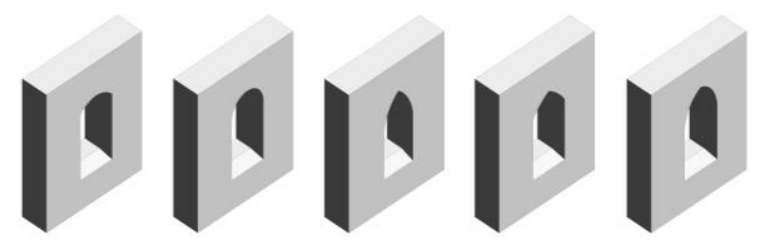

Figure 3. Various examples of round-headed and pointed arch configurations producible with the single parametric opening model.

Although the same opening types appear in several instances across the building their dimensions from instance to instance can vary slightly in section and elevation. The single parametric version allows all size variations present from instance to instance to be compensated for by simply adjusting the given parameters of the model. For example opening type 1 in instance $\mathrm{A}$ has a width of $\mathrm{XA}$, while instance $\mathrm{B}$ of the same opening type has a width of $\mathrm{xB}$; meanwhile the window schedule categorizes both instances as the same opening type (Figure 5). Further benefits are seen through the ability to quickly upgrade all instances of that same opening type at once while still maintaining all of the different size variations present across the multiple instances it is placed. Reference planes and reference lines within the Revit families act as a user-friendly means to adjust the geometry of the opening manually within the Revit family editor by clicking and dragging the reference lines. The parametric values additionally are constantly displayed within the model as numeric values of the opening. Accounting for the long term use of the model, these parameters will remain intact throughout the entirety of the model's lifespan. If a measurement of the opening is recorded differently by hand in the future for example, the Revit user can type in the new measurement and the opening would automatically adjust. Additionally to acquire basic measurements in the future, a user needs only to click on the appropriate opening type to find its measurements.
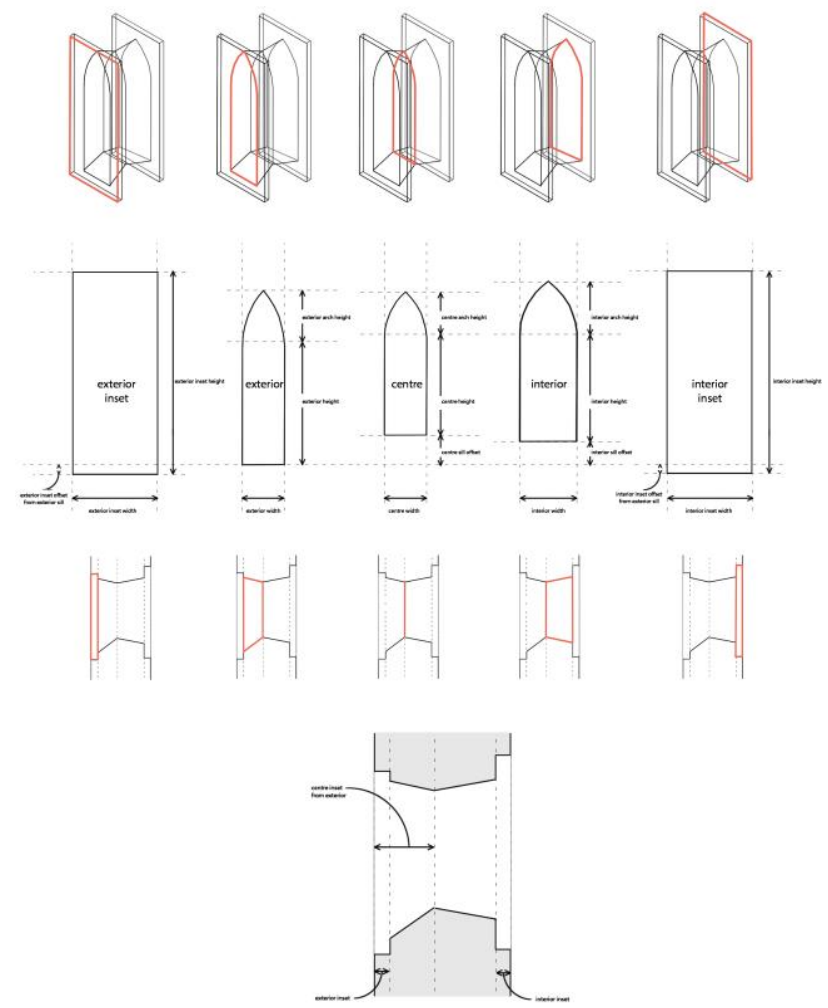

Figure 4. Diagrams representing the series of parametric objects controlled in both section and elevation.

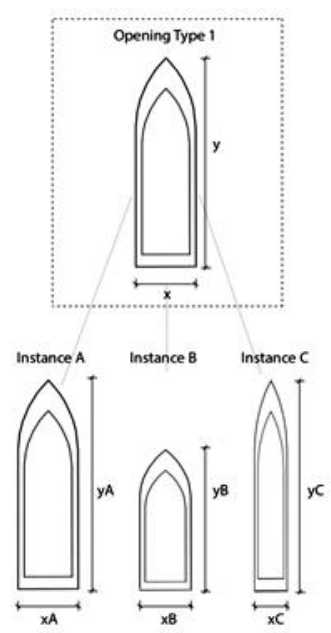

Figure 5. Diagram expressing the capacity for multiple instances of varying scale to be derived from a single typical opening type. By updating the opening type all of its instances are also automatically updated. 
The number of parameters in the model has been reduced to the most fundamental parameters deemed appropriate for accurately recording the scale of each opening type within centimetres of accuracy of the TLS data. The high resolution point cloud from the TLS data proved as an effective template for placing and adjusting the scale of each opening from instance to instance (figure 6). A typical parametric arch opening can compensate for any variability between the same opening types and minor variances in both section and elevation. The parametric capacity of BIM tools allows for an easier, faster, more flexible and a more accurate modelling workflow where slight variations from opening to opening exist. This procedure has also proven effective in that there is a consistent parameter naming format that is carried throughout all of the models. By knowing how to control one model, a modeller is easily able to modify all models using a consistent and familiar method.
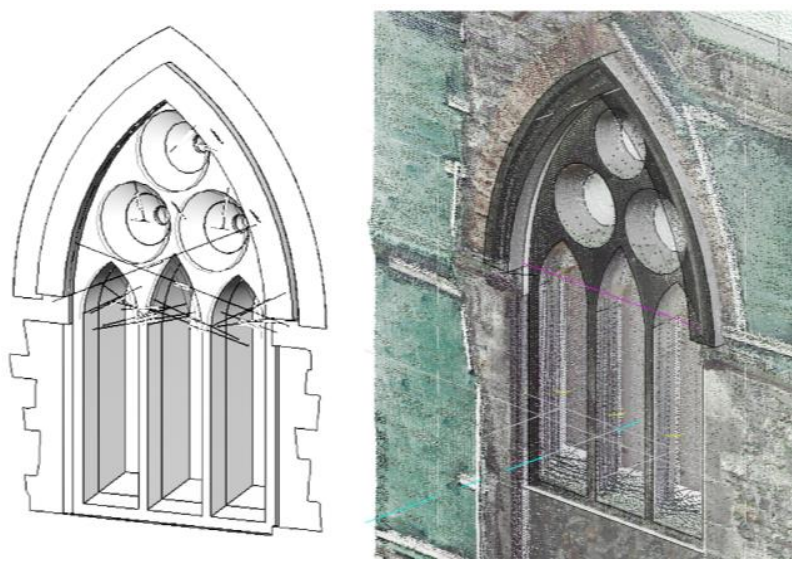

Figure 6. A Parametric model on the left and its insertion based on the TLS data into the Revit wall.

\subsection{Establishing LoD}

In the case of the historic West Block where the building is already highly detailed in its design, defining LoD is a much more difficult task in comparison to modern buildings. Much of the capacity to model higher LoD in existing buildings relies on how it has been documented rather than the capacity of the modelling program to create complex geometries. Point clouds of a poor resolution may not be sufficient enough to produce highly detailed models of architectural elements for example since the density of the points may not be high enough to interpret the form of the element. Because the BIM of the West Block is still a work in progress, effort has been placed into strategically modelling the building for effective long-term use by facility operators by presenting models in three LoD. In the case of the openings, an integral part of proposing three levels of detail is also devising a simple procedure for modifying the models in the future to either add or subtract LoD from the model in cases where the users may opt for more or less information. The (AEC (CAN) BIM Protocol, 2012) in its LoD definition recommends a variety of LoD referred to as component grades (figure 7). These component grades have been used as guidelines to formulate and propose three alternate LoD (figure 8). Which LoD will be the most effective for the future purposes of the West Block BIM is still to be determined, however, it is likely that the final model will consist of multiple LoD.
The following is a description of the AEC (CAN) BIM Protocol's LoD definitions:

Component Grade 1: Is defined as a, "simple place-holder with absolute minimum level detail to be identifiable." (AEC (CAN) BIM Protocol, 2012)

Component Grade 2: Is defined as containing, "relevant metadata and technical information, and is sufficiently modelled to identify type and component materials." (AEC (CAN) BIM Protocol, 2012)

Component Grade 3: Is defined as, "identical to the Grade 2 version if scheduled or interrogated by annotation [and] differs only in 3D representation." (AEC (CAN) BIM Protocol, 2012)

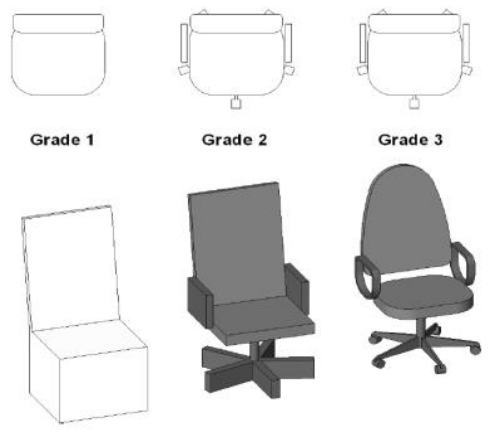

Figure 7. Representation of three levels of detail, referred to as component grades (AEC (CAN) BIM Protocol, 2012).

The translation of these three component grade descriptions into the modelled components of the West Block BIM are defined in the subsequent paragraphs through the presentation of the modelling procedure devised in order to model an opening type at three LoD from the parametric template model. Starting with the basic parametric model as a template, the modelling procedure outlined in the next paragraphs also identifies how the availability of different documentation methods contributes and affects the capacity to model and define the three LoD used in modelling the parametric gothic arch openings.

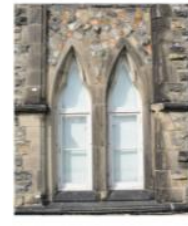

A

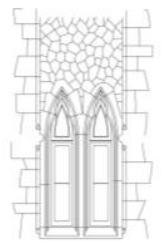

B

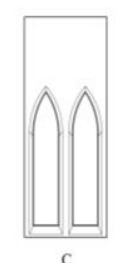

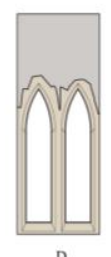

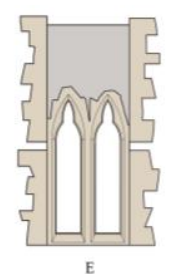

E
Figure 8. Elevations of a common double arch opening type found in the West Block. A-E express the progression of the model starting with a photogrammetric survery (A), 2D CAD drawing (B), model in LoD 1 (C) model in LoD 2 (D), model in LoD 3 (E).

Insertion of the CAD tracings of the photogrammetric survey (LoD 1): A specific 2D trace of any opening instance can be taken from the CAD photogrammetric survey tracing and 
imported into the generic Revit family opening model. Once imported into the family, information of the 2D drawing can be retained in the model regarding the name of the opening instance that the family is based from. Serving as a record of the modelling process, a parametric function can control the visibility of the $2 \mathrm{D}$ tracing so that within the Revit project it can be hidden or displayed according to the user's visualization preference. This allows facility managers to cross-check modelled information directly with the data source that produced it. A new family can be created directly from the parametric template family once the specific $2 \mathrm{D}$ tracing instance has imported (Figure 9). The generic model can be made dimensionally equivalent to the imported data from the $2 \mathrm{D}$ survey tracing by measuring the information displayed within the photogrammetric tracing, or manually adjusting the parameters by clicking and dragging reference lines and planes. A user is able to quickly create a new LoD 1 typical model of an opening instance. This modelling stage is used to produce what has been defined as LoD 1 openings. LoD 1 openings are representational as well as to offer a means of scheduling. They are composed of a series of voids to accurately depict the opening conditions on the exterior face of the wall, the centre of the wall (where the window, jamb etc. is to be placed) and the interior face of the wall. Within each LoD 1 opening family an opening instance from the $2 \mathrm{D}$ survey tracing has been embedded not only as a data reference to create a typical model from but also to assist in upgrading the models into higher levels of detail (LoD 2 and LoD 3). For LoD 1 models, the point cloud assists in positioning the elements within the walls of the BIM. The point cloud also serves as a verification of the dimensions of the typical opening type model across multiple instances. Even at LoD 1, the accuracy of the metric information is consistent with the point cloud.
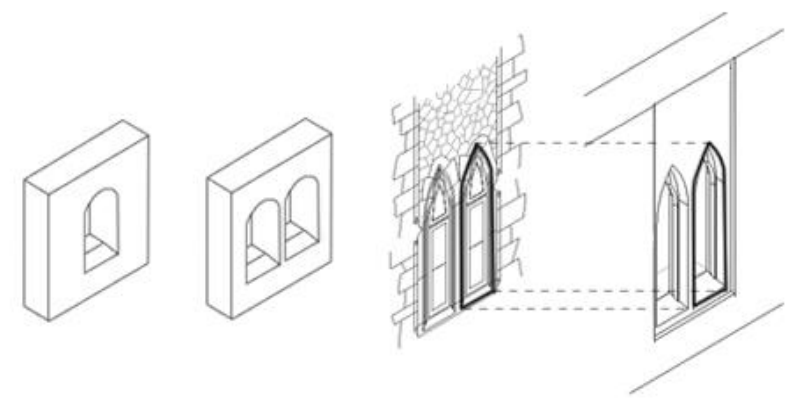

Figure 9. The basic family can be nested into a new family and duplicated in cases where an opening type consists of two arch openings. The new model can then be made to match the $2 \mathrm{D}$ photogrammetric survey traced data by adjusting the parameters, creating a LoD 1 model.

Addition of basic material values (LoD 2): Using the 2D survey tracing as a reference, basic material values of the opening can be added to the model (Figure 10). By aligning the modelled material masses to the already defined parameters, sometimes adding additional parameters in some opening types, all instances of a typical opening type at the LoD 1 stage can be updated with new material information in all instances within the project. If for example there are many instances of an opening type, the model can be updated without losing the scale variability already incorporated into the LoD 1 model. LoD 2 openings are similar in functionality to the LoD 1 openings previously described however; they also include basic material information. The means of scheduling remains consistent with
LoD 1. For LoD 2 models, the point cloud assists in positioning the elements within the walls of the BIM and assists in verifying the position and sizes of the parametric material masses.

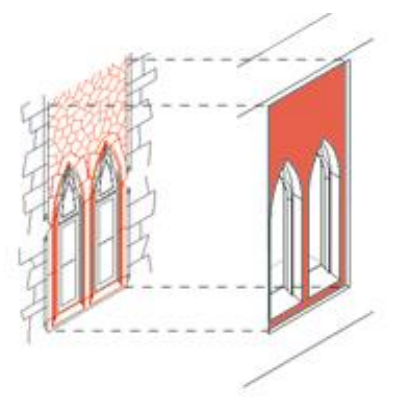

Figure 10. The traced 2D photogrammetric survey data assists further in locating and modeling material masses, creating a LoD 2 model.

Addition of custom elements (LoD 3): LoD 3 openings are further detailed individual families of the LoD 2 family and included geometry of exterior tracery and in some cases stone details that make each window distinguishable from the next. Two methods have been tested to reach a LoD 3 opening model. The first method relies on the accuracy of the $2 \mathrm{D}$ tracing of the photogrammetric survey to model the detailed elements such as the stone tracery around the windows. The second method involves tracing the point cloud data and exporting the 2D lines as a .DWG and then importing them into the opening families. The resolution of the point cloud was not as high as the photos of the photogrammetric survey so in many cases the survey tracing was more reliable as a data source for modelling the geometry of the stone tracery surrounding the LoD 3 models. In LoD 3 openings, each instance has a specific set of geometry that differs from the next which due to the individualization requires an individual model for each instance.

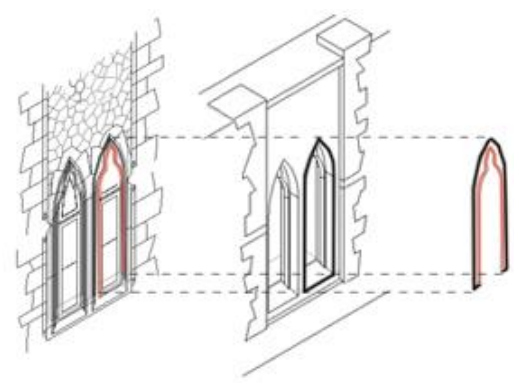

Figure 11. Example of the types of additional details which can be modelled from the 2D photogrammetric survey data by locking masses to both the existing parameters of the model and the $2 \mathrm{D}$ lines of the traced photogrammetric survey.

\section{CONCLUSIONS}

The ability to quickly and freely download parametric three and four dimensional construction assemblies and BIM objects from manufacturer libraries makes BIM an efficient and cost effective tool for the design, construction, and life cycle management of 
new buildings. Recently, BIM has also proven valuable for the documentation of architectural heritage and for the long-term management of heritage assets. Acknowledging an absence of freely available BIM assemblies and objects for heritage buildings, the research discussed in this paper looks to capitalize on the advantages of BIM through the development of a modelling strategy - using TLS data and photogrammetric survey data - to produce 'generic' but parametric Gothic arches that can be modified to satisfy a broad spectrum of unique variations. The ability to address all specific elements in modelling a heritage building — even minor variations in geometry between like elements such as arched openings raises the question of an 'appropriate' level of detail (LoD).

In this case study using the arched openings of the West Block of Canada's Parliamentary Precinct, we explored three LoD based on AEC Canada guidelines. We discovered that the highest LoD — while containing more information - loses some of the parametric benefits of the lower LoD in that the custom elements require each instance of an opening type to have its own separate file, rather than a more generic model with multiple instances. As a result, if one wishes to update all instances of an opening type simultaneously, the highest LoD opening needs to be upgraded individually - a more time consuming operation. Therefore, as in all cases of heritage documentation, it is important to understand what the information will be used for. In the case of building operation and maintenance, a lower level LoD may in fact be more desirable. In the case of documenting unique architectural elements as a posterity recording, the highest possible LoD may be more appropriate. Based on our experience, it is imperative that a detailed documentation strategy be undertaken prior to developing a BIM for heritage documentation, conservation, and management in order to determine and reconcile the LoD of the survey and the model.

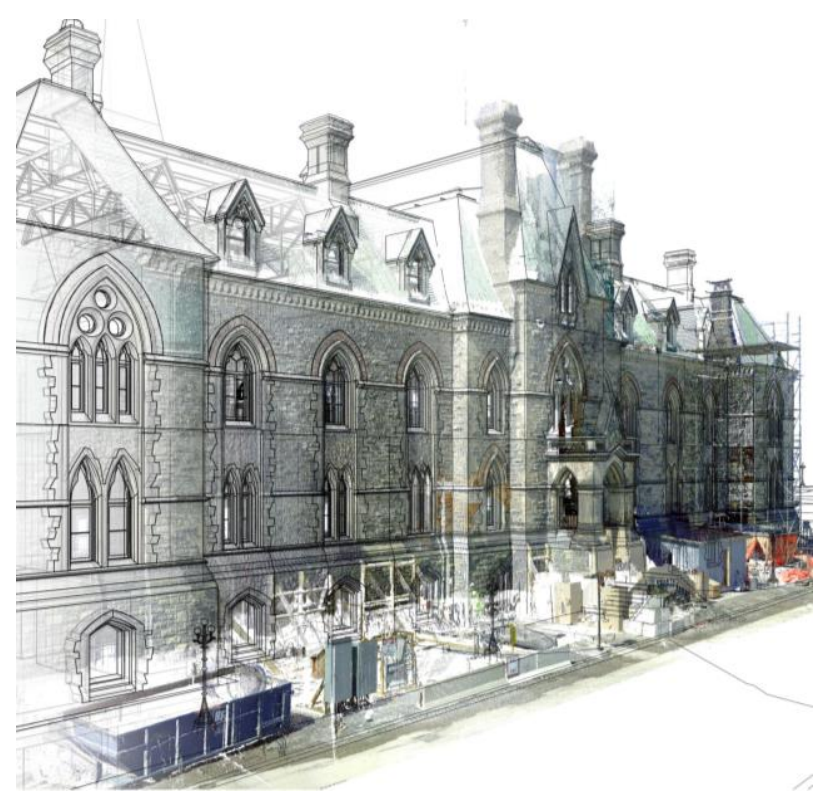

Figure 12. Results of the East Façade (LoD 3) of the BIM with point cloud overlay.

\subsection{References and Selected Bibliography}

American Institute of Architects, 2013. AIA Document G2022013: Project Building Information Modeling Protocol Form.
Autodesk Sustainability Workshop "Design Phases \& BIM Level of Detail", http://sustainabilityworkshop.autodesk.com/ buildings/design-phases-bim-level-detail (1 Mar. 2014).

Baik, A., Boehm, J., Robson, S., 2013. Jeddah Historical Building Information Modelling 'JHBIM' Old Jeddah - Saudi Arabia. International Archives of the Photogrammetry, Remote Sensing and Spatial Information Sciences, Volume XL-5/W2, pp. $73-78$.

Barnes Jr., C. F., 2009. The Portfolio of Villard de Honnecourt: A New Critical Edition and Color Facsimile. Ashgate Publishing Limited, pp. 150.

BIMForum 2013. Level of Development Specification: For Building Information Models, Version: 2013.

Bloxam, M. H., 2006. The Project Gutenberg EBook of The Principles of Gothic Ecclesiastical Architecture, Elucidated by Question and Answer, 4th ed. John Henry Parker, Oxford.

Boulaassal, H., Chevrier, C., Landes, T., 2010. From Laser Data to Parametric Models: Towards an Automatic Method for Building Façade Modelling. EuroMed, Volume 6436 of Lecture Notes in Computer Science, pp. 42-55.

Brumana, R., Oreni, D., Raimondi, A., Georgopoulos, A., 2013. From Survey to HBIM for documentation, dissemination and management of built heritage: The case study of St. Maria in Scaria d'Intelvi. Digital Heritage International Congress (DigitalHeritage), Volume 1, pp. 497-504.

BuildingSMART. http://www.Buildingsmart.org (1 Mar. 2014).

CanBIM AEC (CAN) Designers Committee, 2012. AEC (CAN) BIM Protocol: Implementing Canadian BIM Standards for the Architectural, Engineering and Construction industry based on international collaboration, Version 1.0.

Chevrier, C., Charbonneau, N., Grussenmeyer, P., Perrin, J., 2010. Parametric Documenting of Built Heritage: 3D Virtual Reconstruction of Architectural Details. International Journal of Architectural Computing, Issue 02, Volume 08, pp. 131-146.

Construction Industry Council, BIM Task Group, 2013. Building Information Model (BIM) Protocol: Standard Protocol for use in projects using Building Information Models, first edition 2013.

Corroyer, É., 1893. Gothic Architecture. Macmillan and Co., New York.

Dekeyser, F., Gaspard, F., De Luca, L., Florenzano, M., Chen, X., Leray, P., 2012. Cultural Heritage Recording With Laser Scanning, Computer Vision and Exploitation of Architectural Rules. The International Archives of the Photogrammetry, Remote Sensing and Spatial Information Sciences, Vol. XXXIV, Part 5/W12, pp. 145-149.

Dore, C., Murphy, M., 2013. Semi-automatic modelling of building facades with shape grammars using historic building information modelling. ISPRS International Archives of Photogrammetry, Remote Sensing and Spatial Information Science, Vol. XL 5/W1, pp. 473-480. 
Existing Building Information Modelling (eBIM). http://www.ebim.co.uk/ (1 Mar. 2014).

Garagnani, S., Manferdini, A. M., 2013. Parametric Accuracy: Building Information Modelling Process Applied to the Cultural Heritage Preservation. The International Archives of the Photogrammetry, Remote Sensing and Spatial Information Science, Vol. XL-5/W1, pp. 87-92.

Havemann, S., Fellner, D.W., 2004. Generative Parametric Design of Gothic Window Tracery. The 5th International Symposium on Virtual Reality, Archaeology and Cultural Heritage, VAST.

Hichri, N., Stefani, C., De Luca, L., Vernon, P., 2013. Review of the "As Built BIM" Approaches. International Archives of the Photogrammetry, Remote Sensing and Spatial Information Sciences, Volume XL-5/W1, pp. 107-112.

Ludwig, M., Herbst, G., Rieke-Zapp, D., Rosenbauer, R., Rutishauser, S., Zellweger, A., 2013. The Advantages of Parametric Modeling for the Reconstruction of Historic Buildings. The Example of the in War Destroyed Church of St. Catherine (Katharinenkirche) in Nuremberg. International Archives of the Photogrammetry, Remote Sensing and Spatial Information Sciences, Volume XL-5/W1, pp. 161-165.

Plowman Craven. http://www.plowmancraven.co.uk/ (1 Mar. 2014).

Public Works and Government Services Canada, 2014. "Rehabilitation of the West Block", http://www.tpsgcpwgsc.gc.ca/collineduparlement-parliamenthill/batir-

building/ouest-west/eo-wb-eng.html (1 Mar. 2014).

Oreni, D., 2013. From 3D Content Models to HBIM for Conservation and Management of Built Heritage. ICCSA Part IV, LNCS 7974, pp. 344-357.

Young, C. A., 1995. The Glory of Ottawa: Canada's First Parliament Buildings. McGill-Queen's University Press. 